\title{
Radioprotection by curcumin: A mini review
}

\author{
P Sruthi ${ }^{1,2} \&$ M M Naidu ${ }^{* 1,2}$ \\ ${ }^{1}$ Academy of Scientific and Innovative Research (AcSIR), Ghaziabad - 201 002, Uttar Pradesh. \\ ${ }^{2}$ Department of Spice and Flavour Science, CSIR-CFTRI, Mysuru - 570 020, Karnataka. \\ *E-mail:mmnaidu@cftri.res.in
}

Received 20 April 2021; Revised 07 June 2021; Accepted 14 June 2021

\begin{abstract}
Ionizing radiations are detrimental to the biological system. Exposure to ionizing radiations results in many chronic diseases including cancer. It may cause dysfunctions to almost all organs of the body depending on the total dose, duration and site of irradiation. Apart from its bad effect, radiotherapy is now extensively used for the treatment of several kinds of cancers. Still, the key disadvantage in the procedure is that normal cell, in the surrounding area of the tumor, also receiving radiation doses similar to the tumor, leads to undesirable side effects and risk to patients. Curcumin has been found to protect harmful effects of ionizing radiation. So, it can be beneficial during radiotherapy of cancer. Curcumin helps to kill tumor cells effectively by enhancing the effect of radiation. It also protects normal cells against the harmful effects of radiation. Pre clinical studies are expected to lead to clinical trials to prove the potential of this age-old golden spice for treating cancer patients. This review summarizes the protective effect of curcumin against harmful radiations.
\end{abstract}

Keywords: curcumin, radiation, radioprotection, tumor cells

\section{Introduction}

Medicinal plants are source of numerous organic compounds with therapeutic values. Turmeric (Curcuma longa L.) is typical of the herbaceous plant with thick and fleshy rhizomes that characterize the family Zingiberaceae (Govindarajan 1980). Turmeric contains a wide range of phytochemicals namely, curcuminoids, zingiberene, curcumenol, triethylcurcumin, turmerin, and turmerones (Chattopadhyay et al. 2004).
Curcumin (70-75\%), demethoxycurcumin $(15 \%)$, bisdemethoxycurcumin $(10 \%)$ are the curcuminoids of commercially available turmeric (Shehzad et al. 2003). The value of the turmeric products is based on their curcuminoids content (Merina 2003). Curcumin content in turmeric is approximately $2-6 \%$. This compound was first isolated in 1815, and the structure defined in 1910 as diferuloylmethane with a molecular formula $\mathrm{C}_{21} \mathrm{H}_{20} \mathrm{O}_{6}$ (Figure 1) (Aggarwal et al. 2007; Balaji \& Chempakam 2010). 
Curcumin, the wonder compound, has been widely investigated in recent years due to its high medicinal potential. Several studies indicate that curcumin shows antiinflammatory, anti-angiogenic, antioxidant, wound healing and anti-cancer effects (Maheshwari et al. 2006; Naidu et al. 2009). Curcumin inhibits cancer cell survival and proliferation and induce apoptosis without promoting the development of side effects (Salem et al. 2014). It can scavenge oxygen free radicals, inhibit lipid peroxidation, and protect the cellular macromolecules, including DNA from oxidative damage (Kalpana \& Menon 2004; Polasa et al. 2007). Evidences from literature prove that curcumin exhibit powerful radioprotective activity. Several studies have suggested that curcumin was able to protect healthy cells during radiotherapy (Jagetia 2007).

\section{Effects of ionizing radiations to the biological system}

High energy radiation that has enough energy to remove tightly bound electron, resulting in the formation of ion is named as ionizing radiation. They have adequate energy to remove electrons and ionize atoms of the medium. Major ionizing radiations are X-rays, $\gamma$ - rays, UV rays, $\beta$ particles, $\alpha$ particles and Neutrons. Exposure to ionizing radiations can cause adverse effects to human body.
Effects of ionizing radiations are categorized into two: (i) Higher doses of radiation exposure over a short time result in the short term, or acute effects. (ii) Low doses of radiation exposure over a prolonged time results in long term or chronic effects. Radiations of higher doses can kill the cells and damage tissues and organs, where as low doses of radiation may impair or alter cells. Rapid exposure to body results in Acute Radiation Syndrome (ARS) (Zakariya \& Kahn 2014). Biological system contains more than $80 \%$ water, the predominant effect by which ionizing radiations cause damage to the biomolecules is through radiolysis of water. Peroxide, $\bullet \mathrm{OH}, \bullet \mathrm{H}$ are the reactive oxygen species (ROS) produced when water molecules interact with ionizing radiation (Jagetia 2007; Grant \& Schneider 2000). The hydroxyl radical is a very reactive and oxidizing species. It can react with all cell components (Lin \& Lin-Shiau 2001). Macromolecules are the primary target for the attack of free radicals in the cell (Joe et al. 2004). Free radicals can react with DNA and RNA \& cause structural and functional changes to these biomolecules. If ROS are not effectively scavenged by cellular constituents, they lead to disease conditions (Halliwell \& Gutteridge 1990).

\section{Harmful Effects of Ionizing Radiation}

Harmful effects of radiation exposure vary from simple irritation to deadly diseases including cancer depending upon types, doses

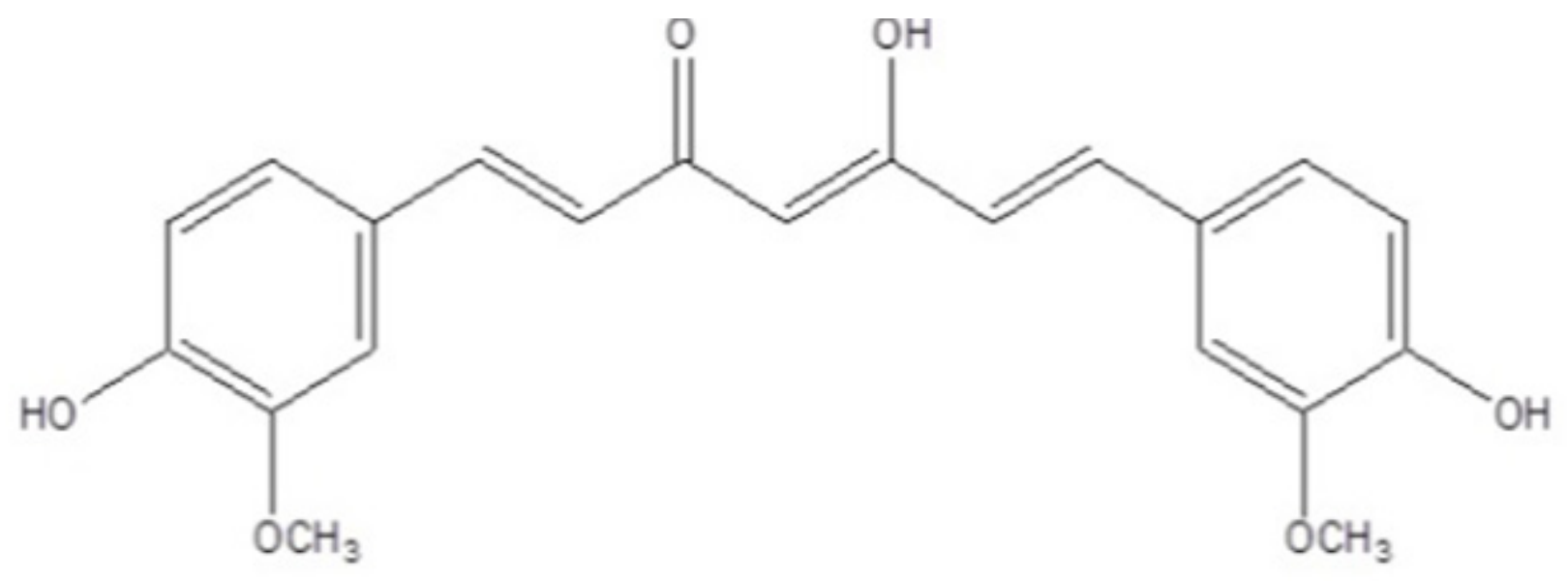

Figure 1: Curcumin structure. 
and duration of radiation. Different factors are responsible for the effects of ionizing radiation. It includes radiation types, doses, time and age of the person. Effects of radiations can be classified into somatic or hereditary effects (Zakariya \& Kahn 2014). Entire body irradiation causes radiation sickness and other syndromes. Radiation syndromes depend upon the doses and duration. More than $50 \mathrm{~Gy}$ radiations are considered as very high dose. Death within minutes to two days is the outcome of exposure of these high doses of radiation and is called central nerve system syndrome. Whole body irradiation to doses between 5 and $10 \mathrm{~Gy}$ cause death of the person within 10 days due to failure of the gastrointestinal tract. If the exposure dose is between 2 to 10 Gy, the death of exposed individual takes up to thirty days. The major reason for this is the damage of bone marrow, resulting in symptoms like fatigue and ulcer of mouth and pharynx. Radiation burns affect soft tissues of skin. Skin injuries vary depending upon duration and doses of radiation. Severe injuries may affect bone and muscles also (Barabanova 2002). Harmful effects of radiation exposure vary from simple irritation to deadly diseases including cancer depending upon types, doses and duration of radiation. Birth anomalies are also an important effect of radiation exposure. Damages to organs are also seen as a result of local body irradiation depending upon radiation doses. In our body, some of the organs are considered as radio resistant and some are radiosensitive. Brain, thyroid, bone, pituitary, adrenals, muscles, and liver are radio resistant. Bone marrow, lymphoid organs, and reproductive organs are radiosensitive (Jagetia 2007). Radiation exposures have the potential to cause genetic effects. But radiation-induced genetic changes are comparatively less in human population than other species.

\section{Therapeutic Uses of Ionizing Radiation}

Ionizing radiations are now used as a powerful tool in the medical field. Mainly used for cancer treatment, radiation therapy is used before surgery to "reduce" the size of the tumor to enable surgical removal. After surgery, radiation helps to destroy any cancer cells that were not removed by surgery. Radiotherapy and chemotherapy can also be used together to efficiently treat the cancer. Radiation therapy is a valuable remedial method in the treatment of different types of tumors. But the effectiveness of this therapy is restricted by its instant and delayed side effects (Grdina et al. 2002). Major disadvantage of radiotherapy is normal cell, near to the tumor receiving radiation doses may result in adverse side effects, increasing the risk of secondary cancers.

Protective effect of curcumin against radiationinduced damage

Radio protective dietary molecules can be effectively and safely used in patients undertake radiotherapy. Patients might tolerate dietary agents better than other synthetic radio protective drugs (Holt et al. 2005). It is better to use natural agents with low cytotoxicity and high antioxidant and anti-inflammatory activities for preventing radiation-induced side effects (Riley 1994). Hence, studies on natural agents provide another important tag to curcumin as an effective radioprotectant. Curcumin administration in cancer patients is claimed to destroy the tumor cells efficiently by improving the benefits of radiation.

\section{Effects of curcumin against gamma radiation}

Gamma rays have much higher energy and are very penetrating. Studies show that $\gamma$-radiation cause many detrimental effects on living systems. Several studies are conducted to understand the radioprotection by curcumin against the harmful impact of $\gamma$-radiation. Radio protective value of curcumin was tested using yeast cells. The result showed that $\gamma$-radiation induced damage in normal yeast cells can be protected by curcumin intake of $1-, 10-$, or $100-\mathrm{mM}$ concentration. A study involving rad 52 mutants also showed that curcumin helps to reduce radiation induced DNA damage (Nemavarkar et al. 2004). A study in mice 
shows that Protein kinase C (PKC) activity is increased in mice liver cytosol subsequent to 5 Gy irradiation. In contrast, treatment of liver cytosol with curcumin reduced the radiationinduced PKC activity (Varadkar et al. 2001). Radiation-induced lipid peroxidation in rat liver microsomes was found to be inhibited by curcumin and tetrahydrocurcumin (THC). Increase in radiation doses also increases lipid peroxidation. But curcumin help to decrease this lipid peroxidation (Jagetia 2007; Khopde et al. 2000).

Another study in mice showed that, intake of 5,10 , and $20 \mathrm{mg} / \mathrm{kg}$ body weight curcumin remarkably decrease the frequencies of micronucleated polychromatic erythrocytes (MPCEs) exposed to $1.15 \mathrm{~Gy}$ or $0.05 \mathrm{~Gy} / \mathrm{s} \gamma$-radiation at 24, 30, and $48 \mathrm{~h}$ post-irradiation (Abraham et al. 2003). Treatment with $400 \mu \mathrm{mol}$ of curcumin decreased the radiation induced chromosomal aberrations and micronuclei in polychromatic erythrocytes of mice exposed to 1.5-3 Gy $\gamma$-radiation. Curcumin helps to protect rat from radiation-induced mammary tumorigenesis without any side effects, indicating that it can be used as an efficient chemoprevention agent (Inano et al. 1999). Another study showed that $200 \mathrm{mg} / \mathrm{kg}$ body weight of curcumin administration reduced the radiationinduced mucositis in rats (Rezvani \& Ross 2004). Curcumin also shows a defensive action on $\gamma$-radiation induced DNA damage and lipid peroxidation in cultured human lymphocytes, and it displays a modulatory effect on $\gamma$-radiation induced cellular damage in primary culture of isolated rat hepatocytes. This study proved that curcumin can be used as an effective radio protector during radiation therapy (Hooper et al. 1987).

Glyoxalase enzyme system has an important role that regulates cell division and differentiation and glyoxalase $\mathrm{I}$ is radiosensitive (Sharma \& Kale 1993). In a study, 0-6 Gy radiation exposure to mice liver elevated the activity of glyoxalase I. In contrast, glyoxalase II activity was inhibited after irradiation. 5, 25 and $50 \mathrm{mg} / \mathrm{kg}$ body weight of curcumin intake before exposure to radiation and also resulted in the restoration of glyoxalase I activity to a control level (Kale 1996). Other studies showed that curcumin decreased acute and chronic skin toxicity in mice indicating that curcumin has a defensiveeffect on radiation-induced cutaneous injury in mice. It reduces the inflammatory and fibrogenic cytokines in irradiated tissues mainly in the early phase after radiation (Okunieff \& Ding 2006). Kapoor et al. (2001) studied protective effect of curcumin against radiation induced protein damages. Low doses of radiation induced chromosomal damage can be protected by different combination of dietary antioxidant including $\beta$-carotene, $\alpha$-tocopherol, ascorbic acid and curcumin (Sharma et al. 1994). Curcumin shows radiosensitizing effect in prostate cancer cell line3. Treatment with curcumin helps to defend against radiation-induced micronuclei in rat bone marrow erythrocytes (Bagheri et al. 2018). A study on lung cancer in mice revealed that antioxidant effect in the lung can be increased by curcumin. In vitro studies showed that curcumin increases heme oxygenase 1 (HO-1) in primary lung endothelial and fibroblast cells and prevent reactive oxygen species (ROS) by radiation exposure. This leads to the increase in antioxidant defense (Lee et al. 2010).

In another study, radiation-induced lipid peroxidation was inhibited by curcumin. Curcumin was incorporated into microsomes during ultracentrifugation. During postirradiation incubation, lipid peroxidation can be effectively inhibited by curcumin in a concentration-dependent manner (Sreejayan et al. 1992). Pre-treatment with curcumin protects the cell from $\gamma$-radiation induced damage by inhibiting peroxidation of membrane lipids and free radicals induced DNA strand break formation (Srinivasan et al. 2006).

Radiation dermatitis has been exhibited by more than $95 \%$ of breast cancer patients. Clinical trial of thirty breast cancer patients revealed that oral intake of $6 \mathrm{~g}$ curcumin decreased the complications of radiation dermatitis in breast cancer patients (Ryan et 
al. 2013). Human serum albumin (HAS) is an important transport protein of the circulatory system. $\gamma$ - radiation cause degradation of secondary structure of HAS. Presence of curcumin offered protection to retain the structural properties of this protein. The protective effect of curcumin was significantly higher in low $\gamma$-radiation doses (Kar et al. 2017). Shabeeb et al. (2020) reported that curcumin administration prevented $\gamma$-radiation induced injury to the skin by elevation of activities of anti-oxidant enzymes. Curcumin also shows protective effect on ionizing radiationinduced cataractogenesis in rats (Ozgen et al. 2012). Oral administration of curcumin shows protective effect on $\gamma$ - irradiation induced hepatic toxicity in rats through regulation of the therapeutic targets CYP2E1, Nrf2, and $\mathrm{NF}-\kappa \mathrm{B}$, through organizing the miR-122 and miR-802 gene expression (Essawy et al. 2020). Curcumin protects radiation-induced liver damages in rats by reducing oxidative stress and inflammation through the modulation of NF- $\kappa B$ pathway. Hence, curcumin treatment before radiotherapy can be helpful to prevent hepatocytic damage (Li et al. 2021).

\section{Curcumin and $U V$-radiation}

Exposure of excessive sunlight is the primary way to enter ultraviolet radiation in the human body. UV A, B and C are the common types of ultraviolet radiation. UV-B cause acute inflammation and nonmelanoma skin cancer in the epidermis (Grossman \& Leffell 1997). Excessive sunlight exposure leads to increase of acute inflammation along with erythema, edema, and immunosuppression. This leads to skin cancer (Granstein \& Matsui 2004). UV irradiation causes p53 mutation, which leads to the formation of precancers, and precancerous lesions converted in to carcinoma in situ and squamous cell carcinoma (SCC) (Jagetia 2007; Ziegler et al. 1994). UV-B causes harmful effect through the formation of reactive oxygen species (ROS) and direct DNA damages (Gruijl 2002). Direct DNA damage or ROS frequently triggers mitogen-activated protein kinases (MAPKs) that are involved in proliferation and survival of the cells (Rhee 1999; Torres \& Forman 2003).

UV radiation is one of the major physical stress factors that affect human skin. UV-A radiation induces photoaging. Liu et al. (2018) reported that curcumin shows protective effect against UV-A irradiation induced photoaging in human dermal fibroblasts. Curcumin inhibits UV radiation-induced skin cancer in $\mathrm{SKH}-$ 1mice, and this antioxidant help to reduce skin cancer formation and prolong time to tumor onset (Phillips et al. 2013). The treatment of rats with curcumin in a dose of $5 \mathrm{mg} / \mathrm{kg}$ body weight prior to ultra violet $C$ irradiation resulted in protection against harmful effect of radiation, while higher doses of curcumin (25 \& $50 \mathrm{mg} \mathrm{kg}^{-1}$ body weight) had less protecting effect (Sharaf et al. 2012).

\section{Mechanism of radioprotection}

Radiation leads to the formation of harmful reactive oxygen species. That involves a chain of events causing DNA damages including strand breakage, base damages and cross link formation. Double strand breaks are very harmful and it may kill the cells. Several mechanisms are involved in the radioprotective effect of curcumin (Jagetia 2007). The assumed mechanisms of radioprotection by curcumin are shown in (Table 1).

Table 1. Curcumin action during radioprotection

\begin{tabular}{ll}
\hline Up regulation & Down regulation \\
\hline DNA repair & Cell cycle \\
Antioxidant activity & DNA damage \\
GSH, GST, SOD, CAT & PKC, MAPK, NO \\
Free radical scavenging & Lipid peroxidation \\
GSH, GST, mRNA & \\
\hline
\end{tabular}

The main leading mechanisms of radioprotection involve free radical scavenging and the upgradation of cellular antioxidants by curcumin in irradiated systems. Glutathione 
transferase (GST), catalase, glutathione peroxidase, superoxide dismutase (SOD), and their mRNAs are increased by curcumin. Increase in glutathione (GSH) and sulfhydryl groups and reduction in lipid peroxidation can also help in radioprotective activity (Jagetia 2007). Curcumin also inhibits the activation of protein kinase C (PKC), mitogen-activated protein kinase (MAPK), and nitric oxide (NO). It also helps to protect against radiationinduced damage (Subramanian et al. 1994; Joe \& Lokesh 1994).

\section{Radiation and wound-healing}

Radiation exposures are continuously increasing, primarily in the use of radiation against cancer therapeutics and other medical treatments (Shore 1990). When wound tissue exposed to ionizing radiation results in disturbance to the normal healing process. It also leads to longer recovery and other detrimental effects. Wounds exposed to radiation, resulting in slow maturation of granulation tissue, severe inhibition of inflammatory response, reduced collagen and hexosamine synthesis and delayed re-epithelialization (Rudolph et al. 1988). Fibroblasts play a major role in granulation tissue formation and maturation. Fibroblast isolated from the irradiated wound and the wound after total-body irradiation has significantly reduced attachment, adhesion, and colony formation ability and have longer doubling time compared to non-irradiated fibroblasts (Song et al. 1997). Studies indicate that topically applied curcumin on wounds has positive effects. Curcumin enhances cutaneous wound healing by granulation tissue formation and synthesis of matrix proteins. Curcumin application has been found to promote re-epithelialization and improves neovascularization. Curcumin intake before irradiation improved the synthesis of collagen, hexosamine, DNA, and nitrate (Jagetia \& Rajanikant 2004a).

Curcumin pretreatment results in decreased wound healing time, improved collagen deposition, increase in fibroblast and vascular density (Jagetia \& Rajanikant 2005). So, curcumin treatment shows profound effect on improving the delayed wound healing. Protective effect of curcumin in woundhealing is due to the increased synthesis of DNA, collagen, hexosamine and nitrite during the healing process in the granulation tissue, which allowed early wound modelling. Wound biopsies examination showed increased fibroblasts, angiogenesis, and collagen deposition. Curcumin treatment has a conductive effect on the irradiated wound and could be a solid healing strategy in tissue repair processes of irradiated wounds (Jagetia \& Rajanikant 2004b).

\section{Challenges of curcumin and its bioavailability}

Although, numerous studies have proved the beneficiary effects of curcumin, there are studies suggesting its ineffectiveness. Curcumin act as a prooxidant by activating molecular oxygen through the reduction of transition metals. Treatment of pBR322 plasmid DNA with curcumin and copper ion resulted in strand break, DNA damage and induction of apoptosis (Yoshino et al. 2004). Banerjee et al. (2008) studied antioxidant and prooxidant activities of curcumin in RBCs hemolysis model. Results showed that curcumin exhibits both antioxidant and prooxidant activity in a concentration dependent manner. Curcumin at higher concentration $(>10 \mu \mathrm{M})$ shows prooxidant behavior (Ahsan et al. 1999).

Despite their potential therapeutic properties, widespread utilization of curcumin is limited by its low bioavailability, low solubility, rapid degradation, high hepatic metabolism and instability in physiological environment (Baek et al. 2017). Poor oral bioavailability is one of the major limitations in clinical application of curcumin. In recent years, efforts have been made to enhance the bioavailability of curcumin by different strategies. Different types of nano formulations including nanoparticles, micelles, liposomes, nanogels and conjugates have been developed to overcome the in vivo delivery of curcumin (Yallapu et al. 2015; Li et al. 2020). 
Nanocurcumin exhibits better therapeutic efficiency due to improved bioavailability and tissue distribution. Lipid nanocarriers and polymer nanocarriers can enhance the bioavailability and solubility of curcumin (Yallapu et al. 2015). Solid lipid nanoparticle can be used as an efficient oral delivery system for curcumin (Ban et al. 2020). Beak et al. (2017) reported that N-carboxymethyl chitosan (NCC) coated curcumin-loaded solid liquid nanoparticle inhibit the rapid release of curcumin in acidic environment and enhance the bioavailability. Bioavailability of curcumin can be increased by encapsulation in sophorolipid coated nanoparticles. Both in vitro and in vivo studies showed that curcumin nanoparticle had higher bioavailability than that of curcumin crystals (Peng et al. 2018).

Nosrati et al. (2020) evaluated the radioprotective effect of curcumin conjugated albumin nanoparticles. These nanoparticles have a high loading capacity and showed sustained release behaviour. Loading of curcumin to BSA not only could increase the chemical stability of curcumin, but also could improve radioprotection efficacy of it against X-ray irradiation. In vitro and in vivo studies proved that BSA-curcumin nanoparticles can be used as a proficient vehicle for improving the potential radioprotective effect of curcumin. Studies state that curcumin can be used as a radioprotective agent and is non-toxic up to 12 $\mathrm{g} \mathrm{day}^{-1}$ in humans.

\section{Conclusion}

Curcumin can be used as a natural radioprotective agent. It protects radiationinduced damage at low doses. Curcumin shows radioprotection against the harmful impact of UV and $\gamma$-radiation in various study systems. Radioprotective effect of this natural agent is due to its free radicals scavenging activity and the elevation of antioxidants, upregulation of mRNAs for GSH, GST, SOD and catalase enzyme. Curcumin also shows radiation-induced wound-healing effect, and it can also be used for the treatment of irradiated wound for quick healing. Pretreatment with curcumin leads to decrease in wound healing time, improved collagen deposition, increase in fibroblast and vascular density.

\section{Acknowledgement}

The authors thank Director CSIR-CFTRI, Mysore for the facilities provided. First author acknowledges CSIR, New Delhi for the award of Junior Research Fellowship.

\section{References}

Abraham S K, Sarma L \& Kesavan P C 1993 Protective effects of chlorogenic acid, curcumin and beta-carotene against gammaradiation-induced in vivo chromosomal damage. Mutat. Res. 303: 109-112.

Aggarwal B B, Sundaram C, Malani N \& Ichikawa H 2007 Curcumin: the Indian solid gold. In: The molecular targets and therapeutic uses of curcumin in health and disease (pp. 1-75). Springer, US.

Ahsan H, Parveen N, Khan N U \& Hadi S M 1999 Pro-oxidant, anti-oxidant and cleavage activities on DNA of curcumin and its derivatives demethoxycurcumin and bisdemethoxycurcumin. Chem. Biol. Interact. 121: 161-175.

Baek J S \& Cho C W 2017 Surface modification of solid lipid nanoparticles for oral delivery of curcumin: Improvement of bioavailability through enhanced cellular uptake, and lymphatic uptake. Eur. J. Pharm. Biopharm. 117: 132-140.

Balaji S \& Chempakam B 2010 Toxicity prediction of compounds from turmeric (Curcuma longa $\mathrm{L}$ ). Food Chem.Toxicol. 48: 2951-2959.

Bagheri H, Rezapour S, Naiafi M \& Motevaseli E 2018 Protection against radiation induced micronuclei in rat bone marrow erythrocytes by curcumin and selenium L-methionine. Iran J. Med. Sci. 43: 645-652.

Ban C, Jo M, Park Y H, Kim J H, Han J Y, Lee K W, Kweon D-H \& Choi Y J 2020 Enhancing the oral bioavailability of curcumin using solid lipid Nanoparticles. Food Chem. 302: 125328. 
Banerjee A, Kunwar A, Mishra B \& Priyadarsini K I 2008 Concentration dependent antioxidant/ pro-oxidant activity of curcumin Studies from AAPH induced hemolysis of RBCs. Chem. Biol. Interact. 174: 134-139.

Barabanova A V 2002 Acute radiation syndrome with cutaneous syndrome. In: Ricks R C, Berger M E \& O'Hara F M(Ed.) The Medical Basis for Radiation Accident Preparedness, The Clinical Care of Victims (pp. 217-224). Parthenon, New York.

Chattopadhyay I, Biswas K, Bandyopadhyay U \& Banerjee R K 2004 Turmeric and curcumin: Biological actions and medicinal applications. Curr. Sci. 87: 44-50.

De Gruijl F R 2002 Photocarcinogenesis, UVA vs. UVB radiation.Skin Pharmacol. Appl. Skin Physiol. 15: 316-320.

Eassawy M M T, Salem A A \& Ismail A F M 2021 Biochemical study on the protective effect of curcumin on acetaminophen and gammairradiation induced hepatic toxicity in rats. Environ. Toxicol. 36: 748-763.

Govindarajan V S 1980 Turmeric- chemistry, Technology, and Quality. Crit. Rev. Food Sci. Nutr.12: 199-301.

Granstein R D \& Matsui M S 2004 UV radiationinduced immunosuppression and skin cancer.Cutis. 74: 4-9.

Grant K L \& Schneider C D 2000 Tumeric. Am. J. Health-Syst. Pharm. 57: 1121-1122.

Grdina D J, Murley J S \& Kataoka Y 2002 Radioprotectants: current status and new directions, Oncology. 63: 2-10.

Grossman D \& Leffell D J 1997 The molecular basis of nonmelanoma skin cancer: A new understanding. Arch. Dermatol.133: 12631270.

Halliwell B \& Gutteridge J M C 1990 Role of free radicals and catalytic metal ions in human disease: an overview. Methods Enzymol. 186: 1-85.

Holt P R, Katz S \& Kirshoff R 2005 Curcumin therapy in inflammatory bowel disease: A pilot study. Dig. Dis. Sci. 50: 2191-2193.

Hooper N I, Tisdale M J \& Thornalley P J 1987
Glyoxalase activity during differentiation of human leukaemic cells in vitro. Leuk. Res. 11: 1141-1148.

Inano $\mathrm{H}$, Onoda $\mathrm{M}$, Inafuku N, Kubota M, Kamada Y, Osawa T, Kobayashi H \& Wakabayashi K 1999 Chemoprevention by Curcumin during the promotion stage of tumorigenesis of mammary gland in rats irradiated with X-rays.Carcinogenesis. 20: 1011-1018.

Jagetia G C 2007 Radioprotection and radio sensitization by curcumin. Adv. Exp. Med. Biol. 595: 301-320.

Jagetia G C \& Rajanikant G K 2004a Effect of various doses of curcumin on the radiation impaired healing of excision wound in mice: A preliminary study. J. Wound Care. 13: 107109.

Jagetia G C \& Rajanikant G K 2004b Role of curcumin, a naturally occurring phenolic compound of turmeric in accelerating the repair of excision wound, in mice wholebody exposed to various doses of $\gamma$-radiation. J. Surg. Res. 120: 127-138.

Jagetia G C \& Rajanikant G K 2005 Curcumin treatment enhances the repair and regeneration of wounds in mice hemi-body exposed to $\gamma$-radiation.Plast. Reconstr. Surg. 115: 515-528.

Jagetia G C \& Reddy T K 2005 Modulation of Radiation induced alteration in the antioxidant status of mice by naringin. Life Sci. 77: 780-794.

Joe B \& Lokesh B R 1994 Role of capsaicin, curcumin and dietary n-3 fatty acids in lowering the generation of reactive oxygen species in rat peritoneal macrophages. Biochim. Biophys. Acta. 1224: 255-263.

Joe B, Vijaykumar M \& Lokesh B R 2004 Biological properties of curcumin cellular and molecular mechanisms of action. Crit. Rev. Food Sci. Nutr. 44: 971-11.

Kale R K 1996 Exploitation of hypoxia for radiation therapy: A lesion from phenothiazines. Med. Hypothes. 47: 107-110.

Kalpana C \& Menon V P 2004 Curcumin ameliorates oxidative stress during nicotine induced lung toxicity in Wistar rats. Ital. J. Biochem. 53: 8286. 
Kapoor S \& Priyadarsini K I 2001 Protection of radiation induced protein damage by curcumin. Biophys. Chem. 92: 119-126.

Kar T, Basak P, Ghosh R K \& Bhattacharyya M 2017 Protective effects of curcumin against gamma ray induced conformational change of human serum albumin. Int. J. Biol. Maromol. 99: 600-607.

Khopde S M, Priyadarsini K I, Guha S N, Satav J G, Venkatesan P \& Rao M N 2000 Inhibition of radiation-induced lipid peroxidation by Tetrahydrocurcumin. Possible mechanisms by pulse radiolysis.Biosci. Biotechnol. Biochem. 64: 503-509.

Lee J C, Kinniry P A, Arguiri E, Serota M \& Kanterakis S 2010 Dietary curcumin increases antioxidant defenses in lung, ameliorates radiation induced pulmonary fibrosis, and improves survival in mice. Radiat. Res. 173: 590-601.

Li W, Jiang L, Lu X, Liu X \& Ling M 2021 Curcumin protects radiation-induced liver damage in rats through the NF- $\kappa$ Bsignaling pathway. BMC complement. Med. Ther. 21: 1-10.

Li Z, Shi M, Li N \& Xu R 2020 Application of Functional Biocompatible Nanomaterial to improve curcumin Bioavailability. Front. Chem. 8: 589957.

Lin J K \& Lin-Shiau S Y 2001 Mechanisms of cancer chemoprevention by curcumin. Proc. Natl. Sci. Counc. Repub. China B. 25: 59-66.

Liu X, Zhang R, Shi H, Li X, Li Y, Taha A \& Xu C 2018 Protective effect of curcumin against ultraviolet A irradiationinduced photoaging in human dermal fibroblasts. Mol. Med. Rep. 17: 7227-7237.

Maheshwari R K, Singh A K, Gaddipati J \& Srimal R C 2006 Multiple biological activities of curcumin: a short review. Life Sci.78: 20812087.

Merina B A 2003 Indigenous Medicinal Plants: their extracts and isolates as a value-added export product. J. Agro. Bios.1: 39-41.

Naidu M M, Shyamala B N, Manjunatha J R, Sulochanamma G \& Srinivas P 2009 Simple HPLC Methods for Resolution of Curcuminoids with Antioxidant Potential. J. Food Sci. 74: 312-318.
Nemavarkar P, Chourasia B K \& Pasupathy K 2004 Evaluation of radioprotective action of compounds using Saccharomyces cerevisiae. J. Environ. Pathol. Toxicol. Oncol. 23: 145-151.

Nosrati H, Danafar H, Rezaeejam H, Gholipour N \& Rahimi-Nasrabadi M 2020 Evaluation radioprotective effect of curcumin conjugated albumin Nanoparticles Bioorg. Chem. 100: 103891.

Okunieff P \& Ding I 2006 Curcumin protects against radiation induced acute and chronic cutaneous toxicity in mice and decrease mRNA expression of inflammatory and fibrogenic cytokines. Int. J. Radiat. Oncol. Biol. Phys. 65: 890-898.

Ozgen S C, Dokmeci D, Akpolat M, Karadag C H, Gunduz O, Erbaş H, Benian O, Uzal C\& Turan F N 2012 The Protective Effect of Curcumin on Ionizing Radiation-inducedCataractogenesis in Rats. Balkan Med. J. 29: 358-363.

Peng S, Li Z, Zou L, Liu W, Liu C \& McClements D J 2018 Enhancement of Curcumin Bioavailability by Encapsulation in Sophorolipid-Coated Nanoparticles: An in Vitro and in Vivo Study. J. Agric. Food Chem. 66: 1488-1497.

Phillips J, Moore-Medlin T, Sonavane K, Ekshyyan O \& McLarty J 2013 Curcumin inhibits UV radiation induced skin cancer in SKH-1 mice. Otolaryngol. Head Neck Surg. 148: 797-803.

Polasa K, Naidu N A, Ravindranath I \& Krishanaswamy K 2004 Inhibition of B(a) $\mathrm{P}$ induced strand breaks in presence of curcumin. Mutat. Res. 557: 203-213.

Rezvani M \& Ross G A 2004 Modification of radiation-induced acute oral mucositis in the rat. Int. J. Radiat. Biol. 80: 177-182.

Rhee S J 1999 Redox signaling, hydrogen peroxide as intracellular messenger.Exp. Mol. Med. 31: 53-59.

Riley P A 1994 Free radicals in biology: Oxidative stress and the effects of ionizing radiation. Int. J. Radiat. Biol. 65: 27-33.

Rudolph R, Berge V, Schneider J A, Fisher J C \& Poolman W L 1988 Slowed growth of cultured fibroblasts from human radiation wounds. Plast. Reconstr. Surg. 18: 669-677. 
Ryan J L, Heckler C E, Ling M, Katz A, Williams J P \& Alice P 2013 Curcumin fur radiation dermatitis: a randomized, double-blind, placebo controlled clinical trial of thirty breast cancer patients. Radiat. Res.180: 34-43.

Salem M, Rohani S \& Gillies E R 2014 Curcumin, a promising anti-cancer therapeutic: a review of its chemical properties, bioactivity and approaches to cancer cell delivery. RSC Adv. 4: 10815-10829.

Shabeeb D, Musa A E, Ali H S A \& Najafi M 2020 Curcumin Protects Against RadiotherapyInduced Oxidative Injury to the Skin. Drug Des. Dev. Ther. 14: 3159-3163.

Sharaf H A, Morsy F A, Shaffie N M \& El-Shennawy A T M 2012 Histological and Histochemical Study on the Protective Effect of Curcumin on Ultraviolet Irradiation Induced Testicular Damage in Albino Rats. J. Cytol. Histol. 3: 159.

Sharma L, Abraham S K \& Kesavan P C 1994 Chromosomal damage by low doses of radiation: protection by combination of dietary antioxidants. Curr. Sci. 66: 861-862.

Sharma R \& Kale R K 1993 Effect of radiation on glyoxalase I and glyoxalase II activities in spleen and liver of mice. Int. J. Radiat. Biol. 63: 233-238.

Shehzad A, Rehman G \& Lee Y S 2003 Curcumin in inflammatory diseases.Biofactors. 39: 69-77.

Shore R E 1990 Overview of radiation-induced skin cancer in humans.Int. J. Radiat. Biol. 57: 809827.

Song S \& Cheng T 1997 The effect of systemic and local irradiation on wound macrophage and repair promoting action of phenytoin sodium.Zhonghua Yi XueZaZhi. 77: 54-57.
Sreejayan N, Rao M N A, Priyadarsini K I \& Devasagayam T P A 1997 Inhibition of radiation induced lipid peroxidation by curcumin. Int. J. Pharm.151: 127-130.

Srinivasan M, Rajendra P N \& Venugopal P M 2006 Protective effect of curcumin on $\gamma$-radiation induced DNA damage and lipid peroxidation in cultured human lymphocytes. Mutat. Res. 611: 96-103.

Subramanian M, Sreejayan R M N A, Devasagyam T P A \& Singh B B 1994 Diminution of singlet oxygen-induced DNA-damage by curcumin and related antioxidants. Mutat. Res. 311: 249-255.

Torres M \& Forman H J 2003 Redox signaling and the MAP kinase pathways. Biofactor. 17: 287296.

Varadkar P, Dubey P, Krishna M \& Verma N C 2001 Modulation of radiation-induced protein kinase $C$ activity by phenolics.J. Radiol. Prot. 21: 361-370.

Yallapu M M, Nagesh P K, Jaggi M \& Chauhan S C 2015 Therapeutic applications of curcumin nanoformulations. AAPS J. 17: 1341-1356.

Yoshino M, Haneda M, Naruse M, Htay H H, Tsubouchi R, Qiao SL, Li W H, Murakami K \& Yokochi T 2004 Prooxidant activity of curcumin: Copper-dependent formation of 8- hydroxy-2'-deoxyguanosine in DNA and induction of apoptotic cell death. Toxicol. InVitro. 18: 783-789.

Zakariya N I \& Kahn M T E 2014 Benefits and Biological Effects of Ionizing Radiation. Sch. Acad. J. Biosci. 2: 583-591.

Ziegler A, Jonason A S, Leffell D J, Simon J A, Sharma H W, Kimmelman J, Remington L, Jacks T \& Brash D E 1994 Sunburn and p53 in the onset of skin cancer. Nature 372: 773-776. 